\title{
High glucose alters the expression of genes involved in proliferation and cell-fate specification of embryonic neural stem cells
}

Received: 7 April 2005 / Accepted: 31 October 2005 / Published online: 1 March 2006

C) Springer-Verlag 2006

\begin{abstract}
Aims/hypothesis: Maternal diabetes induces neural tube defects during embryogenesis. Since the neural tube is derived from neural stem cells (NSCs), it is hypothesised that in diabetic pregnancy neural tube defects result from altered expression of developmental control genes, leading to abnormal proliferation and cell-fate choice of NSCs. Materials and methods: Cell viability, proliferation index and apoptosis of NSCs and differentiated cells from mice exposed to physiological or high glucose concentration medium were examined by a tetrazolium salt assay, 5-bromo-2'-deoxyuridine incorporation, terminal deoxynucleotidyl transferase-mediated dUTP nick end labelling and immunocytochemistry. Expression of developmental genes, including sonic hedgehog $(S h h)$, bone morphogenetic protein 4 (Bmp4), neurogenin 1/2 (Neurog1/2), achaete-scute complex-like 1 (Ascl1), oligodendrocyte transcription factor 1 (Olig1), oligodendrocyte lineage transcription factor 2 (Olig2), hairy and enhancer of split 1/5 (Hes 1/5) and delta-like 1 (Dll1), was analysed by real-time RT-PCR. Proliferation index and neuronal specification in the forebrain of embryos at embryonic day 11.5 were examined histologically. Results: High glucose decreased the proliferation of NSCs and differentiated cells. The incidence of apoptosis was increased in NSCs treated with high glucose, but not in the differentiated cells. High glucose also accelerated neuronal and glial differentiation from NSCs. The decreased proliferation index and early differentiation of neurons were evident in the telencephalon of embryos derived from diabetic mice. Exposure to high glucose altered the mRNA expression levels of Shh,
\end{abstract}

J. Fu $\cdot$ S. S. W. Tay $\cdot$ E. A. Ling $\cdot$ S. T. Dheen $(\bowtie)$

Molecular Neurobiology Laboratory,

Department of Anatomy,

Yong Loo Lin School of Medicine,

National University of Singapore,

Blk MD 10, 4 Medical Drive,

Singapore 117597, Singapore

e-mail: antstd@nus.edu.sg

Tel.: +65-6516-3217

Fax: $+65-6778-7643$
Bmp4, Neurog1/2, Ascl1, Hes1, Dll1 and Olig1 in NSCs and Shh, Dll1, Neurog1/2 and Hes5 in differentiated cells. Conclusions/interpretation: The changes in proliferation and differentiation of NSCs exposed to high glucose are associated with altered expression of genes that are involved in cell-cycle progression and cell-fate specification during neurulation. These changes may form the basis for the defective neural tube patterning observed in embryos of diabetic pregnancies.

Keywords bHLH factors - Bmp4 - Differentiation - Dll1 . Glucose $\cdot$ Neural stem cells $\cdot$ Neural tube defects $\cdot$ Notch . Proliferation $\cdot$ Shh

Abbreviations Ascl1: achaete-scute complex-like 1 . bHLH: basic helix-loop-helix - BMPs: bone morphogenetic proteins - BrdU: 5-bromo-2'-deoxyuridine . DAPI: 4',6-diamidino-2-phenylindole dihydrochloride . Dll1: delta-like 1 - GFAP: glial fibrillary acidic protein . Hes 1/5: hairy and enhancer of split 1/5 - HG: high glucose concentration - MAP2: microtubule-associated protein 2 . Nes: nestin - Neurog 1/2: neurogenin 1/2 - NG2: chondroitin sulphate proteoglycan - NSCs: neural stem cells - NTD: neural tube defect - Olig1: oligodendrocyte transcription factor 1 - Olig2: oligodendrocyte lineage transcription factor 2 - PG: physiological glucose concentration - SHH: sonic hedgehog - STZ: streptozotocin - TUNEL: terminal deoxynucleotidyl transferase (Tdt)-mediated dUTP nick end labelling - XTT: tetrazolium salt

\section{Introduction}

The association between maternal diabetes and the incidence of congenital malformations, including neural tube defects (NTDs), has been well established [1-4]. Although the molecular mechanisms by which maternal diabetes induces NTDs are not known with certainty, it has been suggested that altered expression of genes involved in neural tube development may contribute to the NTD $[2,5$, $6]$. Since the neural tube is derived from neural stem cells 
(NSCs), which are self-renewing, multipotent progenitors, giving rise to different cell types that compose the nervous system, such as neurons, astrocytes and oligodendrocytes, it is possible that maternal diabetes may influence the proliferation and differentiation of NSCs leading to neural tube defects during embryogenesis. The proliferation of NSCs and the sequential appearance of neurons and glia in the neural tube of developing vertebrate embryos are coordinately regulated by various signalling molecules, which include sonic hedgehog (SHH), a member of the hedgehog family of signalling molecules, and bone morphogenetic proteins (BMPs), members of the TGF- $\beta$ superfamily [7-11]. SHH and BMPs regulate positional and temporal specification of neural precursors and the overall shape of the neural tube during development by opposing induction-termination mechanisms $[12,13]$. In addition, several transcription factors with a basic helixloop-helix (bHLH) motif have been implicated in neurogenesis and gliogenesis by controlling the proliferation, specification and differentiation of neural precursors during development. Among them, neurogenin 1/2 (Neu$\operatorname{rog} 1 / 2$, also known as Ngn1/2), hairy and enhancer of split 1/5 (Hes 1/5) and achaete-scute complex-like 1 (Ascll, also known as Mash1) regulate neurogenesis [14-18], while oligodendrocyte transcription factor 1 (Olig1) and oligodendrocyte lineage transcription factor 2 (Olig2) regulate the specification of oligodendrocytes [19]. Hes 1 and Hes 5 are essential factors for Notch/Delta signalling, which regulates the maintenance of undifferentiated cells and functionally antagonises differentiation genes such as Ascll, thus controlling both cell cycle and differentiation $[15,17]$. A recent study reported that Shh promotes the development of neurons and oligodendrocytes from forebrain NSCs by activating the expression of Ascll and Olig2, with a modulation of BMP signalling [20]. Previously, it has been demonstrated that altered expression of Shh and Bmp 4 and their downstream genes is associated with the abnormal patterning of the telencephalon in embryos of diabetic mice [5]. Hence it is hypothesised that maternal diabetes alters the expression of some developmental control genes leading to abnormal proliferation and cell-fate choice of NSCs, thereby resulting in patterning defects during neural tube development. In order to address this, we investigated the effect of high glucose (HG) concentrations on the growth, survival, proliferation and cell-fate specification of NSCs isolated from the telencephalon of embryonic mice, and on the expression of some developmental genes such as Neurog1, Neurog2, Ascl1, Olig1, Olig2, Hes1, Hes5 and delta-like 1 (Dll1) as well as Shh and Bmp4 in NSCs and their differentiated progeny cells.

\section{Materials and methods}

Animals

Insulin-dependent diabetes was induced in 8-week-old female Swiss Albino mice (Laboratory Animals Centre,
National University of Singapore, Singapore) by an i.p. injection of streptozotocin (STZ, $75 \mathrm{mg} / \mathrm{kg}$ body weight; Sigma-Aldrich, St Louis, MO, USA) dissolved in citrate buffer $(0.01 \mathrm{~mol} / \mathrm{l}, \mathrm{pH} 4.5)$ on three successive days. Blood glucose levels were examined 1 week after STZ injection using a Glucometer Elite (Bayer HealthCare, Elkhart, IN, USA). Only those mice with a non-fasting blood glucose level exceeding $16.7 \mathrm{mmol} / 1$ were used as experimental diabetic mice. Timed mating was carried out by placing three to five female mice with one normal male mouse in a cage overnight. Noon on the day on which a copulation plug was observed was counted as embryonic day 0.5 (E0.5). On E11.5, embryos were collected after Caesarean section of pregnant mice anaesthetised with $3.5 \%$ chloral hydrate. The whole embryos were fixed in $4 \%$ paraformaldehyde in phosphate buffer at $4^{\circ} \mathrm{C}$ overnight and cryoprotected with $20 \%$ sucrose in phosphate buffer at $4^{\circ} \mathrm{C}$. Embryos with forebrain malformations from diabetic mice and normal embryos from non-diabetic mice were used as the experimental and control groups, respectively. Frontal sections of $20 \mu \mathrm{m}$ thickness of the developing brain were cut using a cryostat (Leica CM 3050; Leica Microsystems, Nussloch, Germany). All procedures involving animal handling were in accordance with the Principles of Laboratory Animal Care (NIH publication no. 85-23, revised 1985) and guidelines of the Institutional Animal Care and Use Committee, National University of Singapore.

\section{Growth and differentiation of NSCs}

The primary culture of NSCs was prepared from embryos (E13) from Swiss Albino mice as previously described [21, 22]. Briefly, the telencephalon was dissected out from the embryo and mechanically dissociated in DMEM/F12 (1:1; Invitrogen Life Technologies, Carlsbad, CA, USA). The cell suspension was then filtered through a $70 \mu \mathrm{m}$ nylon mesh (BD Biosciences, Bedford, MA, USA), and cells were plated in $75-\mathrm{cm}^{2}$ flasks (Nalge NUNC, Roskilde, Denmark). The serum-free DMEM/F12 medium was supplemented with insulin-transferrin-selenium supplements (Invitrogen), EGF (20 ng/ml; Sigma-Aldrich), and antibiotic-antimycotic solution (Sigma-Aldrich). The cultures were incubated at $37^{\circ} \mathrm{C}$ in a humidified atmosphere of $5 \% \mathrm{CO}_{2}$ and $95 \%$ air. After 5 days in vitro, neurospheres were harvested by centrifugation, dissociated using trypsin and EDTA (Sigma-Aldrich), and replated. After 3-4 days of growth, new neurospheres formed again, and a second passage was performed. Cells were subcultured at least twice before subsequent experiments.

To examine the effects of HG on undifferentiated NSCs, the dissociated cells were cultured for 3 days to form neurospheres in normal physiological glucose concentration (PG) medium, which contained $5 \mathrm{mmol} / \mathrm{l} \mathrm{D}$-glucose, or in $\mathrm{HG}$ medium, containing $30 \mathrm{mmol} / \mathrm{l}$ D-glucose. For differentiation of NSCs, neurospheres in $\mathrm{PG}$ or $\mathrm{HG}$ medium were transferred into PG or HG differentiation medium (containing 2\% fetal bovine serum but no EGF), respectively, in poly-L-ornithine (Sigma-Aldrich)-coated 
flasks or into 24-well plates with poly-L-ornithine-coated coverslips at the bottom of each well, and cultured for 3 days $[22,23]$.

\section{Viability assay}

The tetrazolium salt (XTT) assay allows the quantitative determination of viable cells. XTT is converted into a formazan dye only by metabolically active cells. The quantity of formazan product is directly proportional to the number of viable cells in culture. The XTT assay was performed by using a cell proliferation kit (Roche Applied Science, Penzberg, Germany). For undifferentiated NSCs, dissociated cells were seeded in 96-well plates in a volume of $100 \mu \mathrm{l} \mathrm{PG}$ or HG medium per well at a density of $3.5 \times 10^{5} / \mathrm{ml}$. After $24 \mathrm{~h}$ or 3 days of incubation, cells were incubated for another $4 \mathrm{~h}$ with addition of $50 \mu \mathrm{l}$ XTT labelling mixture $(0.3 \mathrm{mg} / \mathrm{ml})$. Absorbance $(490-650 \mathrm{~nm})$ was measured using a scanning multiwell spectrophotometer. To determine the viability of differentiated cells derived from NSCs, approximately 300 neurospheres grown in $\mathrm{HG}$ or PG medium for 3 days were transferred into each well of poly-L-ornithine-coated 96-well plates with $100 \mu \mathrm{l} \mathrm{PG}$ or HG differentiation medium. XTT assays were performed after the cells were induced to differentiate for $24 \mathrm{~h}$ or 3 days by the same method as described above. Each experiment was replicated five times. Results were expressed as means $\pm \mathrm{SD}$ of absorbance of cells cultured for 1 and 3 days in PG or HG medium, respectively. The absorbance ratio between day 3 and day 1 in each group (PG or HG) was calculated. The absorbance ratio indicates the relative increase of viable cells after 3 days of growth.

Proliferation index by BrdU labelling and immunostaining

Cell proliferation was evaluated by measuring the incorporation of 5-bromo-2'-deoxyuridine (BrdU3; Sigma-Aldrich) during the S-phase of the cell cycle. For NSCs, neurospheres were transferred into 24-well plates with poly-L-ornithine-coated coverslips and incubated with BrdU $(10 \mu \mathrm{mol} / \mathrm{l})$ for $2 \mathrm{~h}$. For the detection of BrdU incorporation in differentiated cells, neurospheres were allowed to differentiate in differentiation medium for 3 days before they were incubated with $\operatorname{BrdU}(10 \mu \mathrm{mol} / \mathrm{l})$ for $2 \mathrm{~h}$. Both NSCs and differentiated cells were fixed with $4 \%$ paraformaldehyde for $20 \mathrm{~min}$, treated with $2 \mathrm{~mol} / \mathrm{l} \mathrm{HCl}$ at $37^{\circ} \mathrm{C}$ for $30 \mathrm{~min}$, blocked with $2 \%$ normal goat serum for $30 \mathrm{~min}$, and incubated with anti-BrdU monoclonal antibody $\left(1: 1,000\right.$; Sigma-Aldrich) overnight at $4^{\circ} \mathrm{C}$. Subsequently, the cells were incubated with goat antimouse secondary antibody conjugated to FITC (1:200; Chemicon, Temecula, CA, USA) for $1 \mathrm{~h}$ at room temperature and counterstained with propidium iodide (5 $\mu \mathrm{g} / \mathrm{ml}$; Sigma-Aldrich) for counting the total cell number. The percentage of BrdU-positive cells was determined by randomly scoring 20 neurospheres for each group (PG or HG medium) in each independent experiment $(n=5)$. The proliferation index is expressed as mean $\pm \mathrm{SD}$ of the percentage of BrdU-positive cells.

For immunocytochemistry, fixed neurospheres and differentiated cells were incubated with appropriate primary antibodies overnight at $4^{\circ} \mathrm{C}$. Primary antibodies used were rabbit polyclonal anti-microtubule-associated protein 2 (MAP2; 1:500; Chemicon) for neurons, rabbit polyclonal anti-chondroitin sulphate proteoglycan (NG2; 1:300; Chemicon) for immature oligodendrocytes, rabbit polyclonal anti-glial fibrillary acidic protein (GFAP; 1:1,000; Chemicon) for astrocytes, and mouse monoclonal antiNestin (1:500; Chemicon) against the intermediate filament marker for neural precursor cells. Subsequently, cells were incubated with FITC-conjugated secondary antibodies (1:200; Chemicon) for $1 \mathrm{~h}$ at room temperature, counterstained with 4',6-diamidino-2-phenylindole dihydrochloride (DAPI; $300 \mathrm{nmol} / \mathrm{l}$; Molecular Probes, Eugene, OR, USA) and visualised using an Olympus BX51 fluorescent microscope or Olympus FV500 confocal microscope. The percentage of neurons, oligodendrocytes and astrocytes in relation to the total cell number was determined in 20 random fields under a $\times 20$ objective for each group in five independent experiments.

For analysis of cell proliferation in the telencephalon of embryos, pregnant mice from the control and diabetic groups ( $n=3$ in each group) were injected i.p. with BrdU (100 mg/kg body weight) $2 \mathrm{~h}$ before the embryos were collected by Caesarean section. Double immunofluorescence labelling was carried out for the localisation of neurons and BrdU-positive cells. Frozen sections through the forebrain were cut, rinsed in phosphate buffer $(\mathrm{pH} 7.4)$, incubated with $2 \mathrm{~mol} / 1 \mathrm{HCl}$ at $37^{\circ} \mathrm{C}$ for $30 \mathrm{~min}$, blocked in $5 \%$ normal goat serum and mouse Ig blocking reagent (Vector Laboratories, Burlingame, CA, USA) for $1 \mathrm{~h}$, and incubated with mouse anti-BrdU monoclonal antibody (1:500) and rabbit anti-MAP2 polyclonal antibody (1:500; Chemicon) overnight at room temperature. Subsequently, sections were incubated in Cy3-conjugated goat antimouse IgG (1:200; Chemicon) and FITC-conjugated goat anti-rabbit IgG (1:200; Chemicon) for $1 \mathrm{~h}$, counterstained with DAPI and mounted with fluorescent mounting medium (DAKO, Carpentaria, CA, USA). Finally, photoimages from the sections were captured on a confocal microscope (Olympus FV500; Olympus, Japan). For quantitative analysis, the numbers of BrdU-labelled cells in the dorsal, ventral and whole telencephalon were counted and the respective percentages calculated. A total of six sections (two each from three embryos) from each group (normal and diabetic) were examined. Results were expressed as means $\pm \mathrm{SD}$ of the percentage of BrdU-positive cells.

\section{Detection of apoptosis by TUNEL assay}

Terminal deoxynucleotidyl transferase (Tdt)-mediated dUTP nick end labelling (TUNEL) assays were performed with an in situ cell death detection kit (Roche), according to 
the manufacturer's instructions. Briefly, neurospheres and differentiated cells were fixed with $4 \%$ paraformaldehyde, then incubated with TUNEL reaction mixture containing the terminal deoxynucleotidyl transferase and FITClabelled nucleotides for $60 \mathrm{~min}$ at $37^{\circ} \mathrm{C}$ in the dark. Cells were rinsed with PBS and incubated with propidium iodide $(5 \mu \mathrm{g} / \mathrm{ml})$ for $10 \mathrm{~min}$ to stain the nuclei of all the cells in culture. The apoptotic cells were counted and the data are presented as the percentage of TUNEL cells relative to the total number of cells.

\section{Quantitative real-time RT-PCR}

Total RNA from NSCs and differentiated cells in PG or HG medium was extracted using an RNeasy mini kit (Qiagen, Hilden, Germany). The reaction mixture containing $2 \mu \mathrm{g}$ RNA, $2.5 \mu \mathrm{mol} / 1$ oligo(dT) primer, $200 \mathrm{U}$ Moloney murine leukaemia virus reverse transcriptase (M-MLV; Promega, Madison, WI, USA), $2 \mathrm{mmol} / 1$ of each dNTP and $5 \mathrm{U}$ RNasin in a total volume of $25 \mu \mathrm{l}$ was incubated for $1 \mathrm{~h}$ at $42^{\circ} \mathrm{C}$ to synthesise cDNAs. For quantitative real-time RT-
PCR, $5 \mu$ laliquots of each cDNA were used in the reaction mixture $(20 \mu \mathrm{l})$ containing LightCycler FastStart DNA Master SYBR Green I, $0.5 \mu \mathrm{mol} / 1$ of each primer in a LightCycler instrument (Roche) as instructed by the manufacturer. Table 1 presents the primers used in this study. The fold change of mRNA was analysed by the $2^{-\Delta \Delta C t}$ method [24].

Statistical analysis The results were analysed by a twotailed Student's $t$ test using Microsoft Excel software and expressed as means $\pm \mathrm{SD}$. Differences were considered as significant at $p<0.05$.

\section{Results}

Effects of HG on proliferation and apoptosis of NSCs and differentiated cells

In the presence of EGF, NSCs derived from embryonic (E13) mouse telencephalon underwent proliferation and formed neurospheres in both PG (5 mmol/l D-glucose) and

Table 1 Primers and thermal profiles for real-time RT-PCR

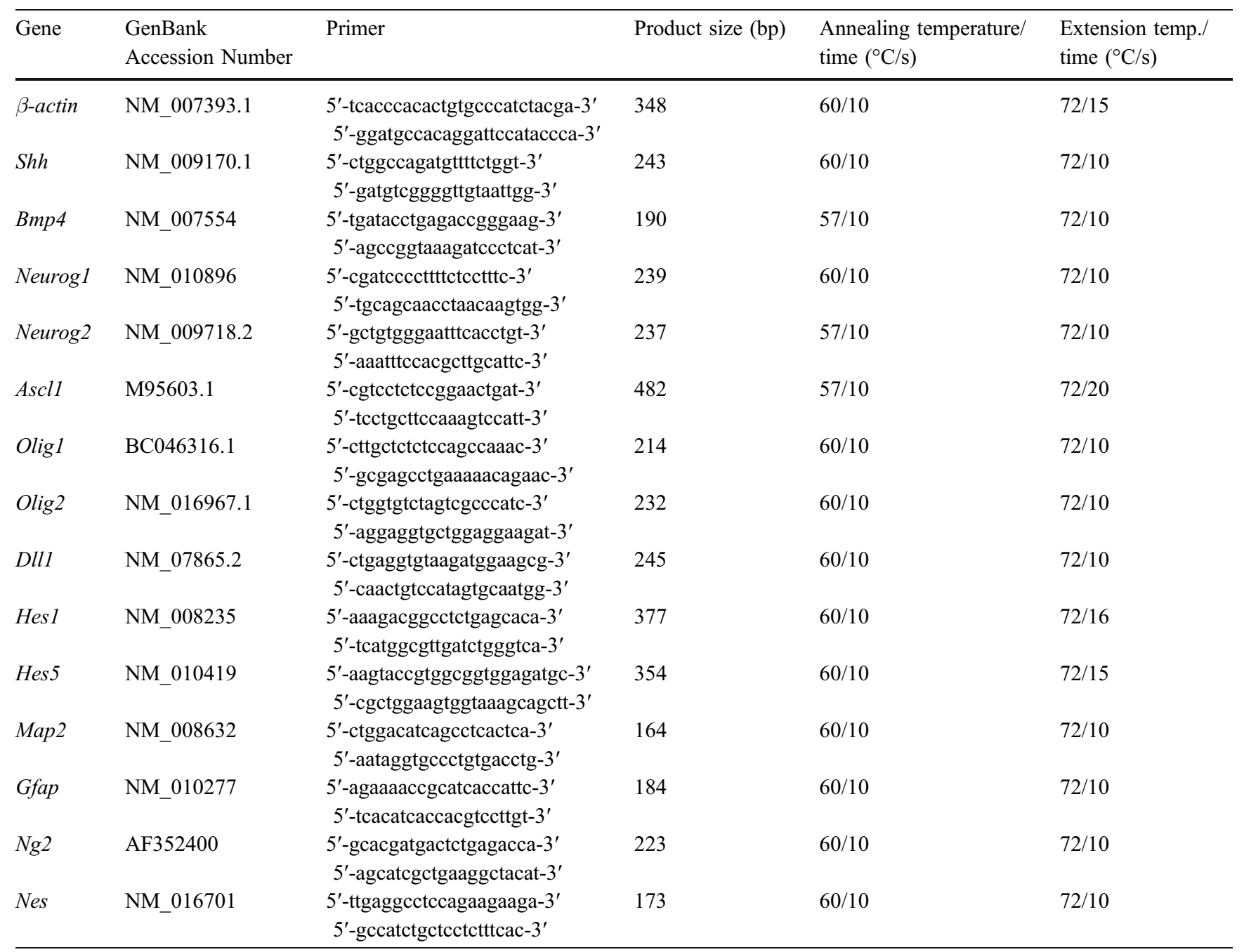


HG (30 mmol/l D-glucose) media. Most of the cells in a neurosphere exposed to either $\mathrm{PG}$ or $\mathrm{HG}$ medium expressed Nestin, an intermediate filament marker of NSCs (Fig. 1a,b). However, the XTT assay showed that after 3 days of growth, the relative increase of viable NSCs in $\mathrm{HG}$ medium was less than that in PG medium $(p<0.05$, Fig. 1c,d). In order to determine whether this variation was due to a decreased proliferation rate or increased incidence of cell death in NSCs cultured in HG medium, the mitotic index by BrdU incorporation and rate of apoptosis by TUNEL were analysed. Pulses of BrdU incorporation in NSCs showed that the percentage of proliferating NSCs in HG medium was decreased compared with that in PG medium ( $30 \pm 8$ vs $42 \pm 5 \%, p<0.01$, Fig. $2 \mathrm{a}, \mathrm{b}, \mathrm{e})$. In contrast, the percentage of apoptotic cells in $\mathrm{HG}$ medium was significantly higher than that in PG medium as demonstrated by TUNEL analysis ( $14 \pm 1$ vs $5 \pm 0.75 \%, p<0.01$, Fig. 2c,d,f).

After 3 days of proliferation in PG or $\mathrm{HG}$ medium, neurospheres were induced to differentiate by withdrawing EGF from the medium. The viability of differentiated cells was measured after 1 and 3 days of incubation. The relative increase of viable cells in $\mathrm{HG}$ medium was the same as those in PG medium (Fig. 3a,b). After 3 days of differentiation, the proliferation index of differentiated cells from NSCs in $\mathrm{HG}$ medium appeared to be significantly decreased compared with that in $\mathrm{PG}$ medium $(0.79 \pm 0.13$ vs $1.10 \pm 0.11 \%, p<0.01$, Fig. $3 c-e)$. However, there was no significant difference in the number of apoptotic cells between the cultures of differentiated cells exposed to PG medium and HG medium (Fig. 3f).

\section{Effects of $\mathrm{HG}$ on the differentiation of NSCs}

The phenotypes of differentiated cells were identified immunocytochemically using antibodies against MAP2 for neurons, GFAP for astrocytes and NG2 for oligodendrocytes. After 3 days of differentiation, all the three types of neural cells were detectable in cultures exposed to both PG and HG media. There were higher proportions of neurons $(31.10 \pm 3.32$ vs $24.53 \pm 2.81 \%, p<0.05)$, oligodendrocytes $(9.03 \pm 2.09$ vs $5.54 \pm 1.74 \%, p<0.05)$ and astrocytes $(45 \pm 1.76$ vs $35 \pm 4.70 \%, p<0.01)$ in differentiated cells exposed to HG medium compared with those in $\mathrm{PG}$ medium (Fig. 4a-g). The mRNA expression of microtubule-associated protein 2 (Map2), chondroitin sulphate proteoglycan $(\mathrm{Ng} 2)$, and glial fibrillary acidic protein (Gfap) was found to be increased and nestin (Nes) was decreased in differentiated cells in HG medium compared with those in PG medium (Fig. 4h), indicating the precocious differentiation of NSCs in $\mathrm{HG}$ medium.

Cell proliferation and neuronal differentiation in the telencephalon of E11.5 embryos of diabetic mice

In order to determine whether the changes in the proliferation of NSCs exposed to HG culture medium mimic the events that occur in the developing neural tube of embryos exposed to maternal hyperglycaemia, we investigated the proliferation and differentiation of neural progenitor cells in the developing telencephalon of E11.5 embryos from diabetic mice. As previously described, the telencephalon in embryos of diabetic mice appeared to be distorted in
Fig. 1 NSCs in neurospheres exposed to medium containing PG (a) $(5 \mathrm{mmol} / \mathrm{l})$ or $\mathrm{HG}$ (b) $(30 \mathrm{mmol} / \mathrm{l})$ immunostained for Nestin (green), a neural precursor cell marker and propidium iodide (red), a general nuclear marker. Most of the NSCs in PG medium and HG medium exhibit Nestin expression. c XTT assays revealed that the number of viable NSCs in both PG (unbroken line) and HG (dashed line) medium is increased from day 1 through to day 3 . However, the relative increase of viable NSCs (d) in HG (filled bar) medium is lower compared with those in PG medium (open bar) and this was shown by the relative ratio between day-3 absorbance and day-1 absorbance. Means \pm SD $(n=5) .{ }^{*} p<0.05$. Scale bar $(\mathbf{a}, \mathbf{b})$ : $50 \mu \mathrm{m}$
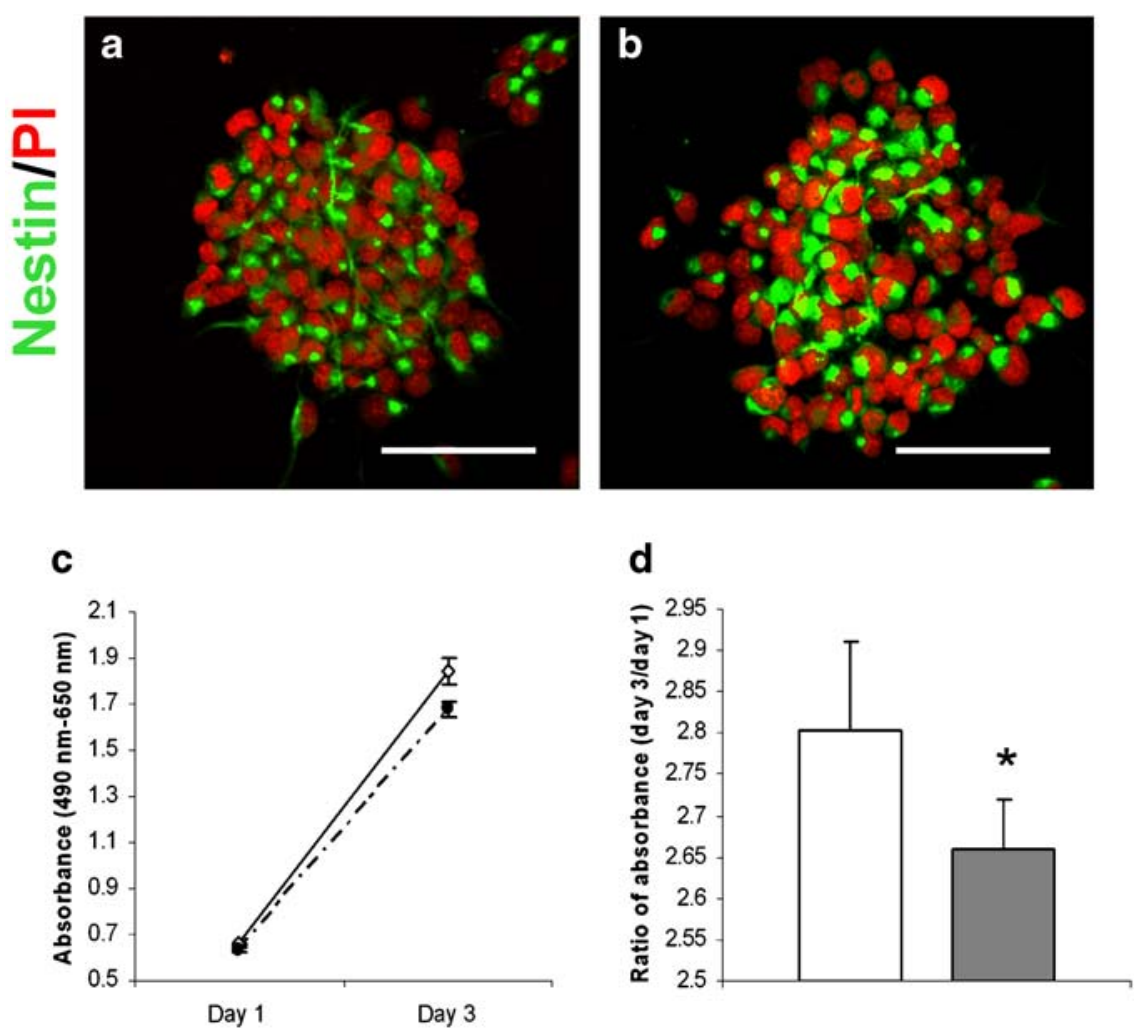
Fig. 2 HG inhibits proliferation and induces apoptotic cell death in NSCs. BrdU-labelled cells in the neurospheres $(\mathbf{a}, \mathbf{b})$ appear in yellow as the BrdU (green) is colocalised with propidium iodide (red). Note the decreased number of BrdU-positive cells in the neurospheres exposed to HG (b, e). The percentage of BrdU-positive cells (e) in neurospheres exposed to HG (filled bar) medium is less in comparison with those in PG medium (open bar). Apoptotic cells in NSCs (arrows in $\mathbf{c}, \mathbf{d}$ ) by TUNEL staining (green) are colocalised with propidium iodide (red). HG (filled bar) increased the percentage of apoptotic cells in NSCs in relation to total cells (f). Means $\pm \operatorname{SD}(n=5) .{ }^{* *} p<0.01$. Scale bar (a-d): $50 \mu \mathrm{m}$
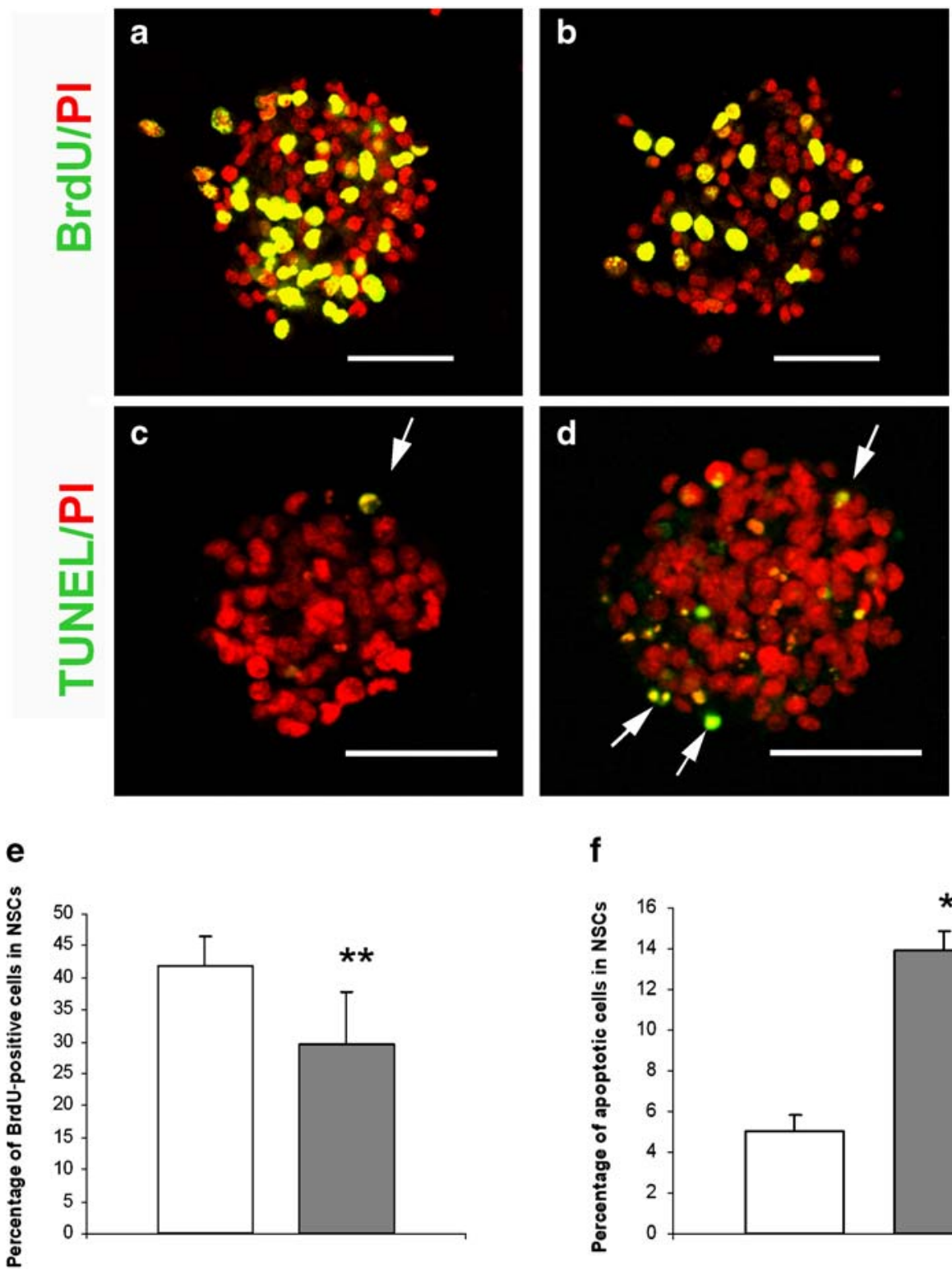

f

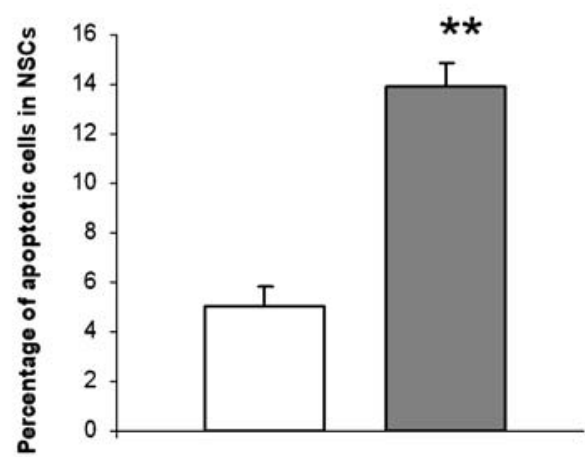

comparison with that of embryos from normal pregnancy (Fig. 5a,d). As the telencephalon is mainly composed of NSCs, the percentage of BrdU-labelled cells in its neuroepithelium can be considered as an index of NSC proliferation in the developing brain. Results of immunohistochemistry showed that the percentage of BrdUpositive cells in the dorsal telencephalon of embryos from diabetic mice was significantly lower than that of controls $(36.86 \pm 1.14$ vs $54.32 \pm 2.28 \%, p<0.01$; Fig. 5 b,e, $\mathrm{g})$. The ventral telencephalon from embryos of diabetic mice showed a significantly higher percentage of BrdU-positive cells than that of controls $(41.75 \pm 1.62$ vs $38.62 \pm 1.34 \%$, $p<0.05$; Fig. $5 \mathrm{c}, \mathrm{f}, \mathrm{g})$. However, the total percentage of BrdUpositive cells in both dorsal and ventral telencephalon from embryos of diabetic mice was lower than that of the controls ( $40.33 \pm 0.87$ vs $43.34 \pm 1.48 \%, p<0.01$; Fig. $5 \mathrm{~g})$. In addition, colocalisation studies showed that there were more MAP2-positive neurons in the whole telencephalon of embryos from diabetic mice than in that of embryos from normal pregnancy (Fig. 5a-f). However, astrocytes and oligodendrocytes were not detectable in situ at this stage.

mRNA expression of signalling molecules and bHLH transcription factors in NSCs and differentiated cells exposed to $\mathrm{HG}$

To determine whether HG in the culture medium influences the expression of Shh, Bmp4, bHLH transcription factors, such as Neurog1/2, Ascl1 and Olig1/2, and members of the Notch signalling pathway, such as Dll1, Hes1 and Hes5, and the mRNA expression levels of these genes in NSCs and differentiated cells were analysed by quantitative realtime RT-PCR. In NSCs cultured in HG medium, $S h h$, Bmp4, Neurog1/2, Ascl1 and Olig1 mRNA expression 
Fig. 3 Effects of HG on viability, proliferation and apoptosis of differentiated cells. a XTT assays at day 1 and day 3 of differentiation. PG, unbroken line; HG, dotted line. b The relative increase of viable cells, which is expressed by the ratio of day-3 absorbance in relation to day-1 absorbance, demonstrates that the relative increase of viable cells in HG medium (filled bar) is the same as those in PG medium (open bar). c, d Confocal images show the colocalisation of BrdU-labelled (red) differentiated cells with MAP2 (green). Only a few BrdU-positive differentiated cells are seen in PG medium (c, arrows) and HG medium (d, arrows). Quantitative analyses demonstrate that the proliferation index (e) is significantly decreased in differentiated cells exposed to $\mathrm{HG}$ (filled bar) and that there is no significant difference (f) in the incidence of apoptotic cell death between the differentiated cells in HG medium (filled bar) and that in PG medium (open bar), as demonstrated by TUNEL analysis. Means $\pm \mathrm{SD}(n=5)$. $* * p<0.01$. Scale bar $(\mathbf{c}, \mathbf{d})$ : $100 \mu \mathrm{m}$ a
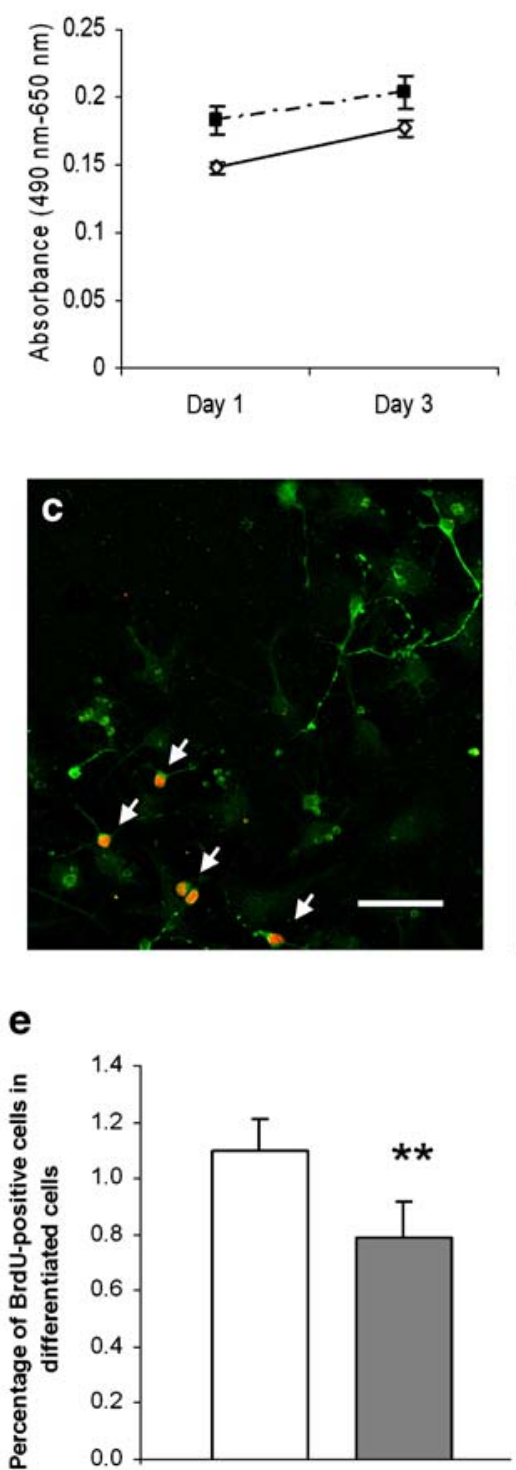

b
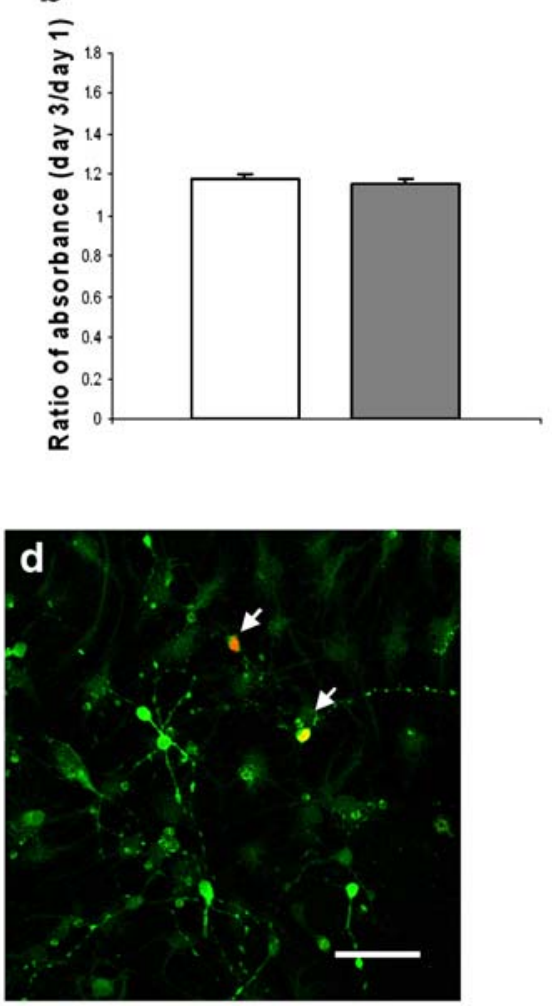

f

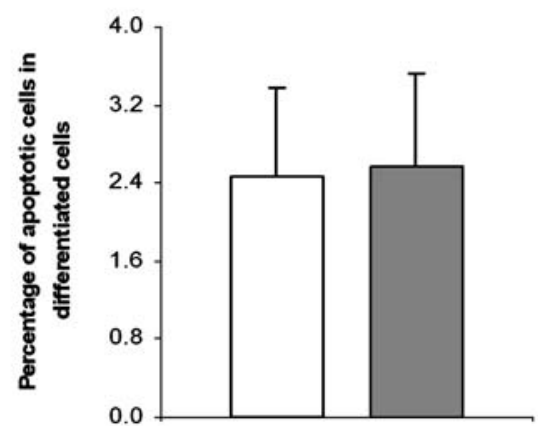

levels were significantly increased, while Dll1 and Hes 1 levels were significantly decreased in comparison with those in PG medium (Fig. 6a). However, there was no significant difference in mRNA expression of Olig2 and Hes 5 in NSCs exposed to PG or HG medium. After 3 days of differentiation, the mRNA levels of Shh, Neurogl, Neurog2, Dll1 and Hes 5 were significantly increased in differentiated cells in HG medium, compared with those in PG medium (Fig. 6b). However, mRNA expression levels of Bmp4, Ascl1, Olig1/2 and Hes1 in differentiated cells appeared to be unaltered by HG concentration.

\section{Discussion}

It has been shown that maternal diabetes induces neural tube defects by altering the expression of various genes involved in the early neural tube patterning that occurs concomitantly with neural induction from NSCs $[2,5,6$, 25]. The proliferation and differentiation of NSCs can be influenced by various maternal factors such as growth factors, cytokines and teratogens, leading to alteration of the cell-fate choice that determines the size, shape and histogenesis of the neural tube $[10,15,25]$. In the present study, HG in vitro impaired the survival and proliferation efficiency and accelerated the early differentiation of NSCs derived from the telencephalon. These changes are associated with the altered expression of developmental control genes, suggesting that NSCs are sensitive to glucotoxicity, which may form the basis for the development of neural tube anomalies in embryos of diabetic pregnancy [5].

A series of experimental studies revealed that HG impaired the cell proliferation in several types of tissues and cell lines. The delay of placental growth in the poorly controlled diabetic pregnancy was correlated with the 
Fig. 4 HG promotes neuronal and glial differentiation of NSCs. Neurospheres grown for 3 days were induced to differentiate in PG $(\mathbf{a}, \mathbf{c}, \mathbf{e})$ or $\mathrm{HG}$ $(\mathbf{b}, \mathbf{d}, \mathbf{f})$ medium. At day 3 of differentiation, cells exhibit immunoreactivity for MAP2, the neuronal marker (a, b arrows, green), NG2, the oligodendrocyte marker (c, d arrows, green), and GFAP, the astrocyte marker (e, f arrows, green). Blue: counterstained by DAPI. g The percentages of MAP2-, NG2- and GFAP-positive cells are increased in differentiated cells in HG medium (filled bars) compared with PG medium (open bars). $\mathbf{h}$ Results of realtime RT-PCR demonstrate that mRNA expression levels of Map2, Gfap, and Ng2 are significantly increased, while mRNA expression level of Nes is significantly decreased, in differentiated cells exposed to HG medium (filled bars) compared with that in $\mathrm{PG}$ medium (open bars). Means \pm SD $(n=5)$. $* p<0.05, * * p<0.01$. Scale bar (a-f): $50 \mu \mathrm{m}$
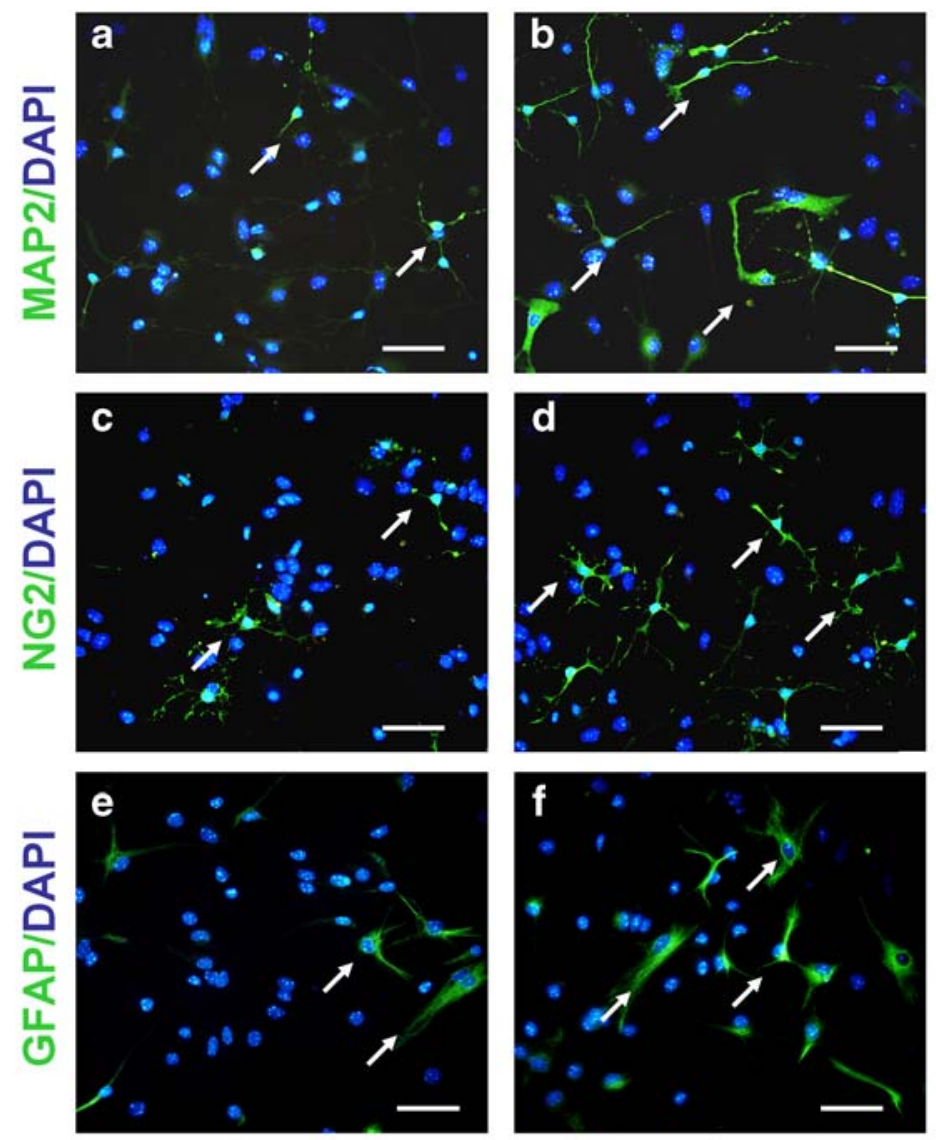

g

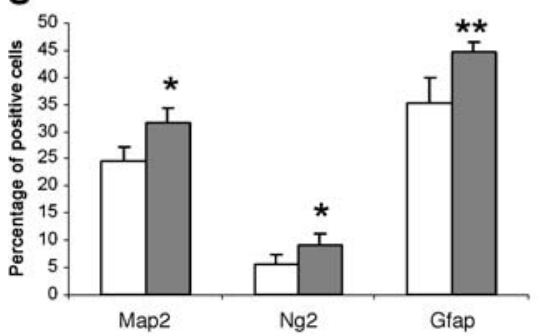

h

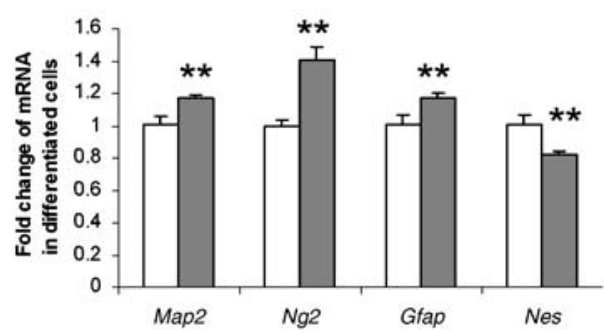

altered proliferation induced by hyperglycaemia [26]. In addition, cell-cycle progression was shown to be decreased in microvessel endothelial cells and mesangial cells exposed to $\mathrm{HG}[27,28]$. In the present study, the proliferation index in NSCs and differentiated cells exposed to HG medium in vitro was found to be decreased. However, in vivo, the proliferation index appeared to be increased in the ventral telencephalon and decreased in the dorsal telencephalon within the forebrain in embryos of diabetic pregnancy, indicating that glucose-responsive local factors could influence the proliferation efficiency. This is possible, as the forebrain morphology is complex and the development of its dorsal and ventral telencephalic neuroepithelium is controlled by a distinct set of signalling molecules and transcription factors, which may exhibit differential response to the glucotoxicity.

In addition, HG medium has been found to alter the expression of several developmental control genes, including Shh and Bmp4, that are involved in cell-cycle progression and apoptosis in the NSCs and differentiated cells. Since the proapoptotic and antiproliferative effects of Bmp4 on various cell types including ventricular zone progenitor cells and sympathetic neuroblasts have been well established [29-31], it is suggested that the HGinduced apoptosis and proliferation arrest in NSCs may be linked to the enhanced expression of Bmp4. However, in differentiated cells exposed to HG, Bmp 4 mRNA expression appeared to be unaltered, indicating that the expression and effects of Bmp4 in response to maternal hyperglycaemia during embryogenesis may vary depending on the location, developmental stage and cell type and its concentration, as well as expression of other regulatory signalling molecules such as SHH [5,31-33]. Shh has been shown to promote the differentiation and survival of neuroepithelial cells by inhibiting apoptotic cell death [34]. Thus, the increased expression of Shh in NSCs and differentiated cells exposed to HG medium could inhibit HG-mediated apoptotic cell death and promote the survival 
Fig. 5 Frontal sections show BrdU and MAP2 staining in the forebrain of embryos (E11.5) from control $(\mathbf{a}-\mathbf{c})$ and diabetic (d-f) mice. Sections are counterstained with DAPI (blue) Compared with controls (a, b), the number of proliferating cells in the dorsal telencephalon of E11.5 embryo from diabetic mice $(\mathbf{d}, \mathbf{e})$ appears to be decreased. However, in the ventral telencephalon, the number of BrdU-positive cells appears to be increased in embryos of diabetic mice $(\mathbf{d}, \mathbf{f})$ compared with that in normal mice (a, c). In addition, there are more MAP2-positive neurons (green) in the telencephalon of embryos from diabetic mice (e, f arrowheads) in comparison with that of controls (b, c arrowheads). Quantitative analysis revealed that the percentage of BrdU-positive cells is significantly decreased in the dorsal telencephalon (b, e arrows; $\mathbf{g})$, increased in the ventral telencephalon (c, $\mathbf{f}$ arrows; $\mathbf{g}$ ), and totally decreased in the whole telencephalon of embryos from diabetic mice (g, filled bars) compared with that of controls (g, open bars). Mean \pm $\mathrm{SD}(n=3) . * p<0.05, * * p<0.01$. Scale bar: a, d $500 \mu \mathrm{m} ; \mathbf{b}, \mathbf{c}, \mathbf{e}, \mathbf{f}$ $100 \mu \mathrm{m} . d t$ Dorsal telencephalon, $e$ eye, $l v$ lateral ventricle, $v t$ ventral telencephalon
DAPI/BrdU/MAP2
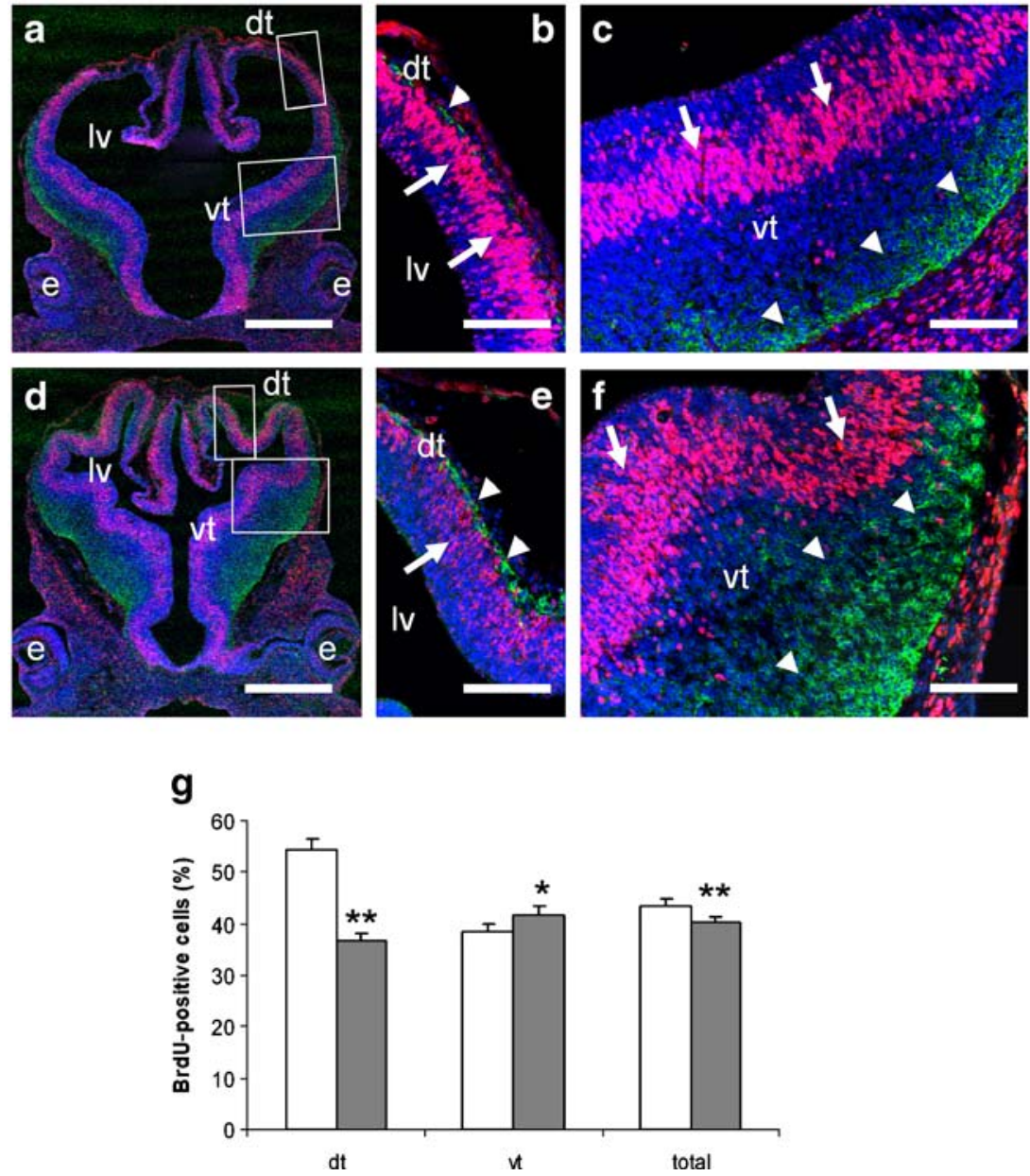

of differentiated cells. Overall, the disturbance in the normal cycle of cell death and proliferation of neural precursor cells and their progenies in the developing neural tube by hyperglycaemia may in turn contribute to the defective patterning and the subsequent complex neural tube anomalies in embryos of diabetic pregnancies.

Besides leading to cell-cycle arrest and apoptosis in NSCs, HG medium also influences the lineage specification of NSCs, increasing the neurogenesis and gliogenesis (including astrocytes and oligodendrocytes) in the early stage of differentiation. Determination of neuron and glia cell fates from NSCs during neural tube development depends on signalling molecules such as SHH and BMPs and their downstream transcription factors such as bHLH factors [35] and homeodomain factors [7]. Several lines of evidence suggest that $S h h$ regulates the development of many neuronal cell types and oligodendrocytes by activating the expression of members of the bHLH factors such as Neurog1, Ascl1 and Olig1 [17, 20, 36-38]. During neurodevelopment, the transient increase in the expression of Neurog1, Neurog2 and Ascl1 (the proneural bHLH factors) results in the initiation of neurogenesis $[14,16$, 18]. In the present study, the increased mRNA expression of Neurog1/2 and Ascll in NSCs and/or differentiated cells exposed to HG could be due to enhanced expression of
Shh, leading to increased neurogenesis. Shh has also been shown to induce two other bHLH transcription factors, Olig1 and Olig2, which promote sequential generation of motoneurons and oligodendrocytes [38-41]. The increased expression of Oligl in NSCs exposed to HG medium appears to be the consequence of enhanced expression of Shh, contributing to increased specification of oligodendrocytes and motoneurons. The unaltered expression of Olig2 in NSCs exposed to HG medium reflects independent roles for Olig1/2 during neurodevelopment. It is suggested that the enhanced Shh expression in the NSCs is the basis for the induced specification of neurons and oligodendrocytes in HG medium.

On the other hand, two other bHLH factors, Hes 1 and Hes5, which inhibit neurogenesis by maintaining progenitors in an undifferentiated proliferative state and by repressing proneural genes such as Ascll and neurogenins [42], also responded to HG. Expression of these genes is regulated by Notch/Delta signalling, which inhibits neuronal differentiation [43]. The decreased mRNA expression of Dll1, a Notch ligand in the NSCs exposed to HG, could have caused downregulation of its downstream gene Hesl, resulting in accelerated neuronal differentiation, possibly by the unopposed action of its target gene Ascll. In contrast, the mRNA expression of Dll1 and Hes5 in the 
Fig. 6 Real-time RT-PCR analysis of mRNA expression levels of genes as indicated in NSCs. a mRNA expression levels of $S h h$, Bmp4, Neurog1/2, Ascll and

Oligl were increased, but those of Dll1 and Hes 1 were decreased in NSCs exposed to HG (filled bars). b Real-time RTPCR analysis data; the expression of Shh, Neurog1/2, Dll1 and Hes 5 mRNA in differentiated cells exposed to $\mathrm{HG}$ (filled bars) was significantly upregulated. Mean values \pm SD $(n=6) . * p<0.05, * * p<0.01$
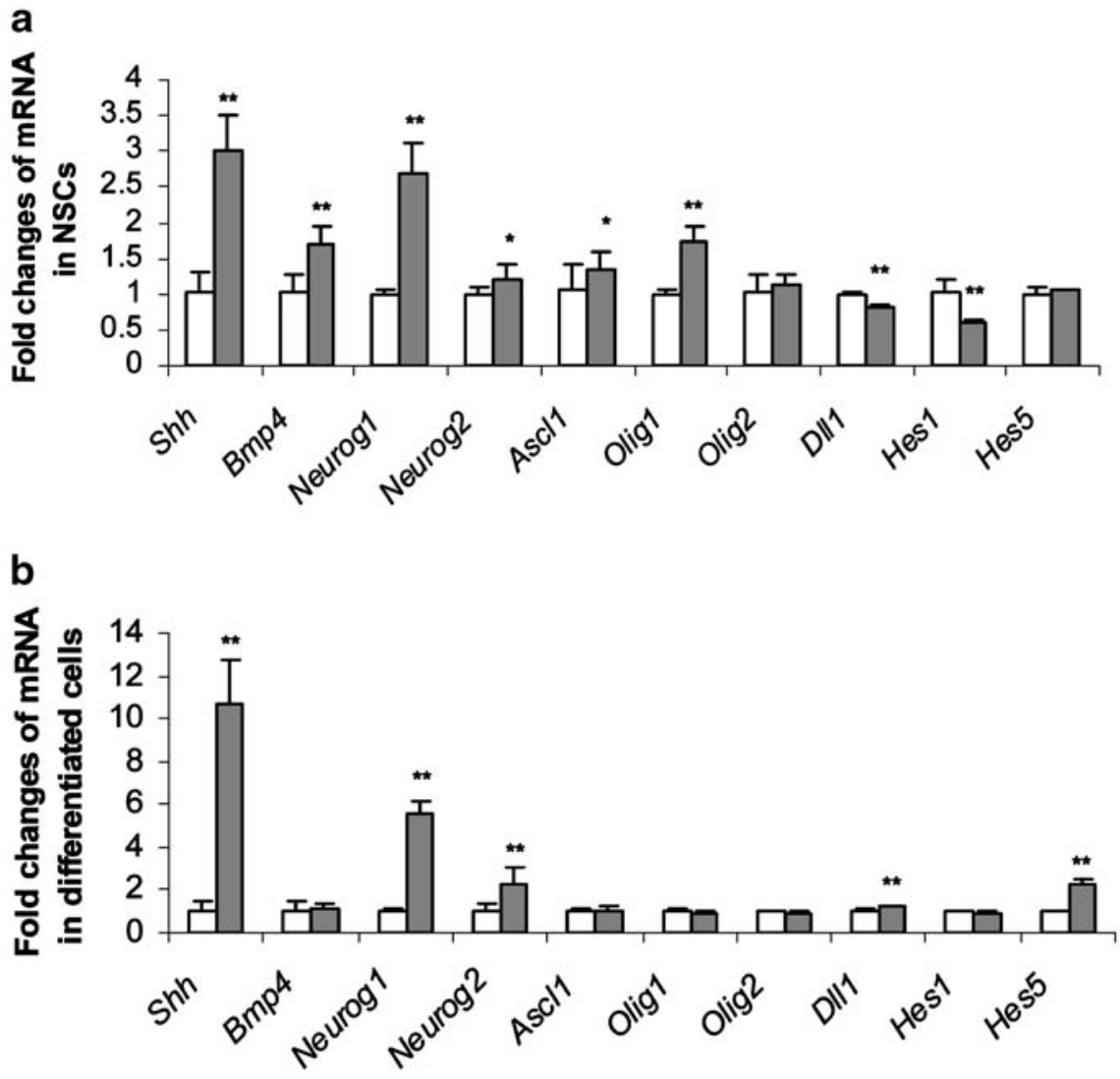

differentiated cells exposed to HG medium was found to be increased, indicating that intrinsic differentiation components may change over time during the development. Moreover, a compensatory mechanism appears to be activated in the differentiating cells to promote astrocytogenesis, which has been shown to be induced by Notch or Hes $5[22,44]$. In the developing neural tube, astrocytes are generated from the dorsal domains where BMP signalling predominates $[45,46]$. BMPs are neurogenic during early stages and gliogenic during late prenatal and early postnatal development. Recently, Bmp4 has been shown to facilitate commitment of NSCs into astrocytes [47, 48], suggesting that the increased mRNA expression of GFAP and increased number of GFAP-immunopositive astrocytes amongst the differentiated cells from NSCs exposed to $\mathrm{HG}$ medium may be mediated by the enhanced expression of Bmp4.

Although the precise mechanisms by which the elevated glucose level alters the expression of a distinct set of genes in NSCs and differentiated cells are not known at present, it is possible that $\mathrm{HG}$ could induce intracellular metabolic disturbances, which may in turn lead to the observed changes in gene expression. In addition, oxidative stress mediated by the glucotoxicity is considered to be the major contributing factor in the altered gene expression, as a similar mechanism has been demonstrated in the inner cell mass of HG-treated mouse blastocysts [49] and in endothelial cells exposed to HG medium [50]. This has been further supported by the finding that hyperglycaemiainduced oxidative stress inhibits the expression of $P a x-3$, a paired box transcription factor essential for the neural tube closure during embryogenesis [51]. It is also possible that there could be a direct effect of $\mathrm{HG}$ on the expression of some developmental genes, as in the process involved in regulation of glucose-responsive genes in the beta-cell or the liver.

In conclusion, this study demonstrates that $\mathrm{HG}$ medium alters the expression of genes that are involved in cell-cycle progression and cell-fate specification in the NSCs and differentiated cells leading to increased neuro- and gliogenesis. We believe that these changes mimic the events that occur during neurodevelopment in embryos of diabetic pregnancies, and that they may form the basis for defective neural tube patterning observed in these embryos. Although the in vitro results obtained in this study may not be directly relevant to human in vivo pathophysiology, they provide some clues, which may be further explored to gain a better insight into the mechanisms underlying the pathogenesis of NTDs associated with diabetic pregnancy.

Acknowledgement This work was supported by a research grant (R-181-000-077-112) from the National University of Singapore, Singapore.

\section{References}

1. Cederberg J, Picard JJ, Eriksson UJ (2003) Maternal diabetes in the rat impairs the formation of neural-crest derived cranial nerve ganglia in the offspring. Diabetologia 46:1245-1251 
2. Fine EL, Horal M, Chang TI, Fortin G, Loeken MR (1999) Evidence that elevated glucose causes altered gene expression, apoptosis, and neural tube defects in a mouse model of diabetic pregnancy. Diabetes 48:2454-2462

3. Hiramatsu Y, Sekiguchi N, Hayashi M et al (2002) Diacylglycerol production and protein kinase $\mathrm{C}$ activity are increased in a mouse model of diabetic embryopathy. Diabetes 51:2804-2810

4. Leunda-Casi A, De Hertogh R, Pampfer S (2001) Decreased expression of fibroblast growth factor-4 and associated dysregulation of trophoblast differentiation in mouse blastocysts exposed to high D-glucose in vitro. Diabetologia 44:1318-1325

5. Liao DM, Ng YK, Tay SS, Ling EA, Dheen ST (2004) Altered gene expression with abnormal patterning of the telencephalon in embryos of diabetic Albino Swiss mice. Diabetologia 47:523-531

6. Phelan SA, Ito M, Loeken MR (1997) Neural tube defects in embryos of diabetic mice: role of the Pax-3 gene and apoptosis. Diabetes 46:1189-1197

7. Jessell TM (2000) Neuronal specification in the spinal cord: inductive signals and transcriptional codes. Nat Rev Genet $1: 20-29$

8. McMahon AP (9-15-2000) Neural patterning: the role of Nkx genes in the ventral spinal cord. Genes Dev 14:2261-2264

9. Morrow T, Song MR, Ghosh A (2001) Sequential specification of neurons and glia by developmentally regulated extracellular factors. Development 128:3585-3594

10. Qian X, Shen Q, Goderie SK et al (2000) Timing of CNS cell generation: a programmed sequence of neuron and glial cell production from isolated murine cortical stem cells. Neuron 28:69-80

11. Sauvageot CM, Stiles CD (2002) Molecular mechanisms controlling cortical gliogenesis. Curr Opin Neurobiol 12:244-249

12. Agarwala S, Sanders TA, Ragsdale CW (2001) Sonic hedgehog control of size and shape in midbrain pattern formation. Science 291:2147-2150

13. Panchision DM, Pickel JM, Studer L et al (2001) Sequential actions of BMP receptors control neural precursor cell production and fate. Genes Dev 15:2094-2110

14. Guillemot F, Joyner AL (1993) Dynamic expression of the murine Achaete-Scute homologue Mash-1 in the developing nervous system. Mech Dev 42:171-185

15. Hatakeyama J, Bessho Y, Katoh K et al (2004) Hes genes regulate size, shape and histogenesis of the nervous system by control of the timing of neural stem cell differentiation. Development 131:5539-5550

16. Lo LC, Johnson JE, Wuenschell CW, Saito T, Anderson DJ (1991) Mammalian achaete-scute homolog 1 is transiently expressed by spatially restricted subsets of early neuroepithelial and neural crest cells. Genes Dev 5:1524-1537

17. Ross SE, Greenberg ME, Stiles CD (2003) Basic helix-loophelix factors in cortical development. Neuron 39:13-25

18. Sommer L, Ma Q, Anderson DJ (1996) Neurogenins, a novel family of atonal-related bHLH transcription factors, are putative mammalian neuronal determination genes that reveal progenitor cell heterogeneity in the developing CNS and PNS. Mol Cell Neurosci 8:221-241

19. Zhou Q, Wang S, Anderson DJ (2000) Identification of a novel family of oligodendrocyte lineage-specific basic helix-loophelix transcription factors. Neuron 25:331-343

20. Yung SY, Gokhan S, Jurcsak J, Molero AE, Abrajano JJ, Mehler MF (2002) Differential modulation of BMP signaling promotes the elaboration of cerebral cortical GABAergic neurons or oligodendrocytes from a common sonic hedgehogresponsive ventral forebrain progenitor species. Proc Natl Acad Sci USA 99:16273-16278

21. Ben Hur T, Rogister B, Murray K, Rougon G, Dubois-Dalcq M (1998) Growth and fate of PSA-NCAM+ precursors of the postnatal brain. J Neurosci 18:5777-5788

22. Grandbarbe L, Bouissac J, Rand M, Hrabe DA, ArtavanisTsakonas S, Mohier E (2003) Delta-Notch signaling controls the generation of neurons/glia from neural stem cells in a stepwise process. Development 130:1391-1402
23. Tropepe V, Sibilia M, Ciruna BG, Rossant J, Wagner EF, van der KD (1999) Distinct neural stem cells proliferate in response to EGF and FGF in the developing mouse telencephalon. Dev Biol 208:166-188

24. Livak KJ, Schmittgen TD (2001) Analysis of relative gene expression data using real-time quantitative PCR and the 2 (Delta Delta C(T)) method. Methods 25:402-408

25. Temple S (2001) The development of neural stem cells. Nature 414:112-117

26. Weiss U, Cervar M, Puerstner P et al (2001) Hyperglycaemia in vitro alters the proliferation and mitochondrial activity of the choriocarcinoma cell lines BeWo, JAR and JEG-3 as models for human first-trimester trophoblast. Diabetologia 44:209-219

27. Abraham NG, Kushida T, McClung J et al (2003) Heme oxygenase-1 attenuates glucose-mediated cell growth arrest and apoptosis in human microvessel endothelial cells. Circ Res 93:507-514

28. Wolf G, Schroeder R, Zahner G, Stahl RA, Shankland SJ (2001) High glucose-induced hypertrophy of mesangial cells requires p27(Kip1), an inhibitor of cyclin-dependent kinases. Am J Pathol 158:1091-1100

29. Glozak MA, Rogers MB (2001) Retinoic acid- and bone morphogenetic protein 4-induced apoptosis in P19 embryonal carcinoma cells requires p27. Exp Cell Res 268:128-138

30. Gomes WA, Kessler JA (2001) Msx-2 and p21 mediate the proapoptotic but not the anti-proliferative effects of BMP4 on cultured sympathetic neuroblasts. Dev Biol 237:212-221

31. Israsena N, Kessler JA (2002) Msx2 and p21(CIP1/WAF1) mediate the proapoptotic effects of bone morphogenetic protein-4 on ventricular zone progenitor cells. J Neurosci Res 69:803-809

32. Furuta Y, Piston DW, Hogan BL (1997) Bone morphogenetic proteins (BMPs) as regulators of dorsal forebrain development. Development 124:2203-2212

33. Mabie PC, Mehler MF, Kessler JA (1999) Multiple roles of bone morphogenetic protein signaling in the regulation of cortical cell number and phenotype. J Neurosci 19:7077-7088

34. Thibert C, Teillet MA, Lapointe F, Mazelin L, Le Douarin NM, Mehlen P (2003) Inhibition of neuroepithelial patched-induced apoptosis by sonic hedgehog. Science 301:843-846

35. Vetter ML, Brown NL (2001) The role of basic helix-loophelix genes in vertebrate retinogenesis. Semin Cell Dev Biol 12:491-498

36. Alberta JA, Park SK, Mora J et al (2001) Sonic hedgehog is required during an early phase of oligodendrocyte development in mammalian brain. Mol Cell Neurosci 18:434-441

37. Ota M, Ito K (2003) Induction of neurogenin-1 expression by sonic hedgehog: its role in development of trigeminal sensory neurons. Dev Dyn 227:544-551

38. Tekki-Kessaris N, Woodruff R, Hall AC et al (2001) Hedgehogdependent oligodendrocyte lineage specification in the telencephalon. Development 128:2545-2554

39. Lu QR, Sun T, Zhu Z et al (2002) Common developmental requirement for Olig function indicates a motor neuron/ oligodendrocyte connection. Cell 109:75-86

40. Nery S, Wichterle H, Fishell G (2001) Sonic hedgehog contributes to oligodendrocyte specification in the mammalian forebrain. Development 128:527-540

41. Zhou Q, Anderson DJ (2002) The bHLH transcription factors OLIG2 and OLIG1 couple neuronal and glial subtype specification. Cell 109:61-73

42. Bertrand N, Castro DS, Guillemot F (2002) Proneural genes and the specification of neural cell types. Nat Rev Neurosci 3:517-530

43. Ohtsuka T, Ishibashi M, Gradwohl G, Nakanishi S, Guillemot F, Kageyama R (1999) Hes1 and Hes5 as notch effectors in mammalian neuronal differentiation. EMBO J 18:2196-2207

44. Tanigaki K, Nogaki F, Takahashi J, Tashiro K, Kurooka H, Honjo T (2001) Notch1 and Notch3 instructively restrict bFGFresponsive multipotent neural progenitor cells to an astroglial fate. Neuron 29:45-55 
45. Briscoe J, Pierani A, Jessell TM, Ericson J (2000) A homeodomain protein code specifies progenitor cell identity and neuronal fate in the ventral neural tube. Cell 101:435-445

46. Briscoe J, Ericson J (1999) The specification of neuronal identity by graded Sonic Hedgehog signalling. Semin Cell Dev Biol 10:353-362

47. Gomes WA, Mehler MF, Kessler JA (2003) Transgenic overexpression of BMP4 increases astroglial and decreases oligodendroglial lineage commitment. Dev Biol 255:164-177

48. Liu SY, Zhang ZY, Song YC et al (2004) SVZa neural stem cells differentiate into distinct lineages in response to BMP4. Exp Neurol 190:109-121
49. Leunda-Casi A, Genicot G, Donnay I, Pampfer S, De Hertogh R (2002) Increased cell death in mouse blastocysts exposed to high D-glucose in vitro: implications of an oxidative stress and alterations in glucose metabolism. Diabetologia 45:571-579

50. Zanetti M, Zwacka R, Engelhardt J, Katusic Z, O'Brien T (2001) Superoxide anions and endothelial cell proliferation in normoglycemia and hyperglycemia. Arterioscler Thromb Vasc Biol 21:195-200

51. Chang TI, Horal M, Jain SK, Wang F, Patel R, Loeken MR (2003) Oxidant regulation of gene expression and neural tube development: insights gained from diabetic pregnancy on molecular causes of neural tube defects. Diabetologia $46: 538-545$ 